\title{
A MEDIDA DE UTILIZAÇÃO DE CAPACIDADE CONCEITOS E METODOLOGIAS*
}

\author{
Carmem Aparecida Feijo ${ }^{* *}$
}

RESUMO O objetivo deste texto é discutir um tema pouco debatido em análises de conjuntura no Brasil que é a precisão das estatísticas sobre o grau de utilização da capacidade produtiva. Partimos da pesquisa de Robin Marris sobre a economia da utilização de capacidade e sugerimos duas interpretações do comportamento das firmas com respeito à tomada de decisões sobre como utilizar a capacidade produtiva. A seguir, avaliamos dois tipos de metodologia para mensuração de capacidade. Concluímos que o conceito de capacidade não apresenta uma definição teórica única e sua mensuração é bastante difícil, implicando que a interpretação dos resultados estimados deve levar em conta as restrições conceituais e metodológicas da variável grau de utilização de capacidade.

Palavras-chave: capacidade produtiva; grau de utilização de capacidade; produto potencial

Código JEL: L16

\section{THE MEASUREMENT OF CAPACITY UTILIZATION: CONCEPTS AND METHODOLOGIES}

ABSTRACT The aim of this paper is to discuss a subject that has not deserved much attention in economic analysis in Brazil: it is about the accuracy of the measure of the degree of capacity utilization. We start with the research by Robin Marris about the economics of capacity utilization and we suggest two interpretations about the behavior of the firm in respect to the decision about how to use productive capacity.

* Artigo recebido em $1^{\circ}$ de novembro de 2005 e aprovado para publicação em 3 de maio de 2006. A autora agradece as valiosas contribuições dos pareceristas anônimos. Erros e imprecisões que porventura persistam continuam sendo de responsabilidade da autora.

** Professora da UFF, pesquisadora CNPq, e-mail: cfeijo@terra.com.br 
Next, we investigate two types of methodology about how to estimate empirically the degree of capacity utilization. We conclude that the concept of capacity does not present a unique theoretical definition and that its empirical estimation is quite difficult, implying that an interpretation of the results should take into account conceptual and methodological limitations of the variable degree of capacity utilization.

Key words: productive capacity; degree of capacity utilization; potential output 


\section{INTRODUÇÃO}

Do ponto de vista das análises de curto prazo, a noção de que a inflação começa a acelerar quando a utilização de capacidade supera determinado patamar é bastante difundida. Além disso, um aspecto freqüentemente observado na realidade contemporânea é a constatação em alguns casos da convivência de situações inflacionárias com subutilização dos fatores produtivos. Essas possibilidades — inflação se acelerando por conta de pressões pelo lado da oferta e a inflação convivendo com a subutilização de recursos produtivos pela insuficiência de demanda agregada - ressaltam a importância do conhecimento sobre excesso ou escassez de capacidade na tomada de decisões de política econômica. A proporção utilizada da capacidade produtiva em determinado momento dá uma dimensão das potencialidades e possíveis pontos de estrangulamento no desempenho futuro da economia.

Uma medida recorrentemente utilizada em análises de conjuntura econômica é o produto potencial. Okun (1962), por exemplo, propôs uma medida de produto potencial como forma de quantificar o desperdício que ocorreria pelo fato de existirem fatores de produção ociosos em determinado momento. Na sua proposta, se a demanda agregada fosse inferior ao nível de demanda de pleno emprego, parte do produto potencial não seria produzida, ou seja, ocorreria um hiato entre o produto potencial e o efetivo. Dessa forma, quantificar o produto potencial ofereceria um guia a políticas de estabilização e seria também um indicador de seu sucesso. ${ }^{1}$

O objetivo deste texto é discutir um tema pouco debatido em análises de conjuntura macroeconômica que é a precisão das estatísticas que suportam a argumentação sobre as decisões de política econômica. Em particular, nossa atenção está voltada para discutir como conceituar e medir o grau de utilização da capacidade produtiva, variável importante na construção e interpretação de medidas de produto potencial. Essa variável, além de ser de difícil mensuração, no Brasil não é produzida por uma fonte estatística oficial, o que implica a ausência de um controle público da qualidade das estatísticas disponíveis. ${ }^{2}$ A despeito das dificuldades técnicas e conceituais para medir a capacidade instalada e da falta de uma referência oficial sobre o grau de ociosidade da capacidade instalada, a estatística grau de utilização de capacidade é uma informação conjuntural relevante na avaliação do potencial de oferta da economia no curto prazo. ${ }^{3}$ 
Do ponto de vista teórico, o estudo do grau de utilização da capacidade justifica-se por pelo menos duas áreas de interesse em economia: como um indicador da demanda agregada a curto prazo e como um dos componentes a influir na tomada de decisões sobre investimentos em capital fixo, a médio e longo prazo. Neste texto damos ênfase à primeira aplicação.

Para introduzir nossa discussão, vamos considerar a representação algébrica da medida de grau de utilização de capacidade expressa como $u=\frac{Y}{Y}$, onde $u$ é a taxa de utilização de capacidade, $Y$ e $Y^{*}$ são respectivamente o produto corrente e o produto a plena capacidade. As diversas metodologias empíricas para estimar o grau de utilização de capacidade visam calcular $u$ e $Y^{*}$.

Conforme mostramos nas próximas seções deste texto, tanto na teoria como na prática, não há uma forma única e de fácil interpretação de como medir o limite de utilização de capacidade. Para discutir esse assunto, sem no entanto termos a pretensão de ser exaustivos, dividimos o presente trabalho em duas partes. Na seçãol apontamos dificuldades teóricas na conceituação de capacidade de produção e indicamos pelo menos duas abordagens distintas de interpretação do comportamento da firma quando avalia seu limite de produção. Nossa sugestão é que a interpretação de resultados sobre grau de utilização de capacidade deve levar em conta uma formulação teórica a respeito de como firmas reagem a estímulos do ambiente econômico. Na seção 2, destacamos aspectos metodológicos da mensuração do grau de utilização de capacidade e produto potencial, ressaltando dificuldades nas estimativas. Uma última seção resume nossas considerações.

\section{A ECONOMIA DA UTILIZAÇÃO DE CAPACIDADE}

A pesquisa empírica conduzida por Robin Marris na Inglaterra nos anos 1950 (Marris, 1964) ${ }^{4}$ sobre como firmas decidem utilizar seu estoque de capital, é uma referência clássica para a mensuração do grau de utilização de capacidade industrial. Essa pesquisa partiu do pressuposto de que empresários, quando planejando seus investimentos, além de escolherem os vários tipos de equipamento, também formam uma idéia do número de horas que o equipamento deve operar regurlarmente. Muitas são as razões que deter- 
minam a taxa planejada de utilização do equipamento, mas uma vez que o investimento foi realizado, a taxa de utilização da planta que será observada dependerá do nível de demanda agregada. Assim, a decisão a respeito da quantidade de equipamento a adquirir irá depender do planejamento das condições de operação da unidade produtiva, mas quanto será efetivamente utilizado estará relacionado a uma administração operacional do dia-a-dia do negócio.

Na visão de Marris, a teoria de utilização de capacidade está diretamente relacionada com a taxa de utilização de capacidade planejada_ao se construir uma planta ou unidade produtiva. Marris oferece uma explicação teórica da razão pela qual firmas operam com excesso de capacidade, ou seja, por que empresários escolhem entre "build a plant designed to produce the required output at a low or moderate reate of utilization, or build a smaller plant designed to produce the same output at a higer rate of utilization (Marris, op. cit.: 5).

A decisão dependerá da interação entre o que chamou de elasticidade de utilização e elasticidade de mecanização. A primeira é obtida pela divisão da variação relativa na taxa de utilização pela variação relativa no salário médio causada pelo aumento na jornada de trabalho. A segunda é calculada a partir da relação entre a variação relativa na produtividade do fator trabalho e a variação relativa nos custos por unidade de mão-de-obra empregada.

Considerando a elasticidade de utilização, que é fortemente influenciada por fatores institucionais, o pressuposto é que as firmas começam com uma dada taxa de salário real e ajustam a ocupação da capacidade posteriormente a seus aumentos. A elasticidade de mecanização será tanto maior quanto mais os ganhos de produtividade decorrentes de um aumento na utilização do equipamento superarem a variação nos custos causada por essa maior utilização. Nesse caso, quanto maior for a elasticidade de mecanização, maior a probabilidade de que um aumento no nível de salário médio seja contrabalançado pela maior utilização do capital.

Mas, pela sua própria natureza, a taxa planejada de utilização da capacidade não pode ser observada objetivamente, pois "it is not at all easy to discover original plans" (op. cit.: 119). A taxa corrente de utilização de capacidade, ou seja, o número de horas que uma planta opera em um determinado período de tempo (uma semana, por exemplo), por sua vez, pode ser in- 
vestigada de forma objetiva. Propõe então, como medida prática do grau de utilização de capacidade, quanto do equipamento disponível de uma planta está sendo operado por turno de trabalho, ${ }^{5}$ assumindo que as firmas incorrem em custos mais altos ao decidirem operar além da capacidade normal, e portanto, ao fazê-lo, esperam receber uma compensação por isso.

Assim, a decisão de operar uma planta a um determinado grau de ocupação pressupõe um conhecimento sobre a capacidade de operação máxima da planta. A forma como essa capacidade máxima é estimada depende das condições previstas em que a planta estará operando quando no seu máximo - em turnos simples de trabalho, em turnos simples com hora extra ou em vários turnos. A resposta a essas condições determina a capacidade máxima de operação da planta, ou seja, o que a firma considera como o máximo de capacidade de produção em um determinado período de tempo.

Conforme reconheceu Marris, essa resposta não é fácil de ser obtida nem inferida, porém é fundamental para o planejamento de pesquisas e a construção de estatísticas que investiguem o grau de utilização de capacidade e para a correta interpretação dos resultados. Ou seja, a interpretação de estatísticas sobre o grau de utilização de capacidade implica considerar a forma como a firma decide sua capacidade máxima de produção, que está também relacionada com a forma como a firma decide sobre a ocupação de sua capacidade.

Nesse sentido podemos sugerir pelo menos duas maneiras de interpretar como as firmas avaliam a capacidade de produção: uma com ênfase em aspectos técnicos de produção e outra com ênfase em aspectos econômicos.

No primeiro caso pode-se tomar como definição de capacidade o montante de produção que tecnicamente pode ser obtido levando-se em conta apenas restrições de engenharia para o pleno funcionamento de um equipamento ou planta industrial. Segundo Leeuw (1962: 833), por exemplo, “the so called 'engineering' approach uses an estimate of the maximum attainable output per machine-year as a measure of annual capacity of a machine". Medir capacidade dessa forma implica conhecer ou convencionar como o equipamento deve ser usado. Por exemplo, a produção máxima que uma planta industrial pode gerar irá variar de acordo com algumas hipóteses assumidas, como a quantidade de turnos de trabalho, quantidade e freqüência de paradas para manutenção, se inclui ou não equipamento fora 
de uso etc. Além disso, é necessário que se considere uma combinação fixa de máquinas e um mix único de produtos, pois alterações em um ou outro conduzirão a resultados distintos do potencial produtivo. ${ }^{6}$

Do ponto de vista teórico, essa definição sugere que a firma é apenas o local que reúne fatores de produção que se combinam de acordo com a tecnologia disponível, ou seja, nada mais é do que uma planta sujeita às leis de rendimento. A firma é retratada como um ator passivo, que toma a tecnologia, preços dos fatores e a capacidade organizacional como dados e reage às mudanças na oferta e demanda por substituição na margem. Aspectos organizacionais e de interação ativa com o ambiente econômico são ignorados, de tal modo que a firma pode ser representada como uma função de produção, cujas entradas são os vários insumos necessários à produção e as saídas, os produtos produzidos por ela. Implícitas nesta abordagem estão hipóteses sobre a eficiência no uso dos fatores de dois tipos. Primeiro, é assumido que a firma opera em sua função de produção que mostra o volume máximo de um produto que pode ser obtido de cada combinação factível dos insumos (capital e trabalho). A falha em operar na função de produção implica desperdício no uso dos insumos. Em segundo lugar, dados os preços dos fatores, assume-se que a firma escolhe a menor combinação de fatores para cada possível nível de produto. ${ }^{7}$

Outra linha de investigação sobre como definir o grau de utilização de capacidade, que chamaremos de definição "econômica" em contraposição ao conceito de "engenharia", procura dar conta de outros aspectos não considerados na abordagem anterior. Nesse caso, considera-se que numa economia de mercado variações no nível de renda e emprego dependem, em grande parte, da tomada de decisões privadas, onde são levadas em conta questões como custos, demanda etc. Quanto e o como produzir a curto prazo para uma firma individual dependem, dadas suas instalações, da relação preço/custo, de sua expectativa quanto ao comportamento da demanda, por exemplo, elementos que passam então a influenciar a estimativa de capacidade.

Podemos sugerir uma forma de explicar como as firmas industriais fixam, na prática, seus preços e, em função destes, determinam a quantidade a ser produzida. Vamos supor que empresários operando num mundo de incerteza dificilmente adotarão o método de "tentativa e erro", para acertar 
qual a quantidade vendida que maximizaria o lucro. Em vez disso, o mais provável é que, conforme Davidson (1978: 35-36),

while recognizing they will not know what level of sales will actually be until near the end of the period, then rational behavior may be associated with choosing some target return on standard volume...Under such conditions, adherence to a conventional mark up over costs which is expected to yield a normal return for some expected or target level of output is readily understandable.

O limite à produção será dado pelo ponto em que o aumento da quantidade não seja mais compensador em termos do retorno esperado.

Vemos assim que implícita nessa interpretação sobre a determinação dos preços para a firma encontra-se uma hipótese de comportamento do empresário que se distancia da idéia de empresário racional, maximizador de lucro, conforme usualmente apresentado em livros texto de microeconomia. Agora, o que está em jogo são as expectativas dos empresários quanto ao comportamento da demanda, que irão influir na decisão de quanto produzir e, conseqüentemente, quanto usar de seu equipamento e instalações. Dentro desse contexto, o conceito de capacidade ganha outra dimensão além das considerações técnicas, dada pelo fato de que o que passa a importar é a avaliação subjetiva dos empresários sobre o que possuem e o que pretendem utilizar. ${ }^{8}$ Devemos acrescentar ainda que a decisão de utilização do capital, baseada nas expectativas do empresário, envolve a noção de Keynes de custo de uso. Segundo Keynes (1936: 69-70),

user cost constitutes the link between the present and the future. For in deciding his scale of production an entrepreneur has to exercise a choice between using up his equipmente now or preserving it to be used later on. ${ }^{9}$

Em suma, considerando que a conceituação de capacidade é pouco precisa pela natureza da varíavel, é necessário associá-la à forma como as firmas decidem até que ponto os empresários estão dispostos a usar o equipamento que consideram disponível, em função de suas expectativas de retorno. A explicitação dessa hipótese de comportamento permite, por um lado, qualificar a variação da produção (se restringida ou facilitada pela menor ou maior margem de ociosidade do equipamento) e, por outro, fornece elementos para inferências sobre ampliações de capacidade a médio ou longo 
prazo. Do ponto de vista de análises de conjuntura e de diagnósticos de política econômica, essas considerações enfatizam a importância de formalizar as hipóteses sobre o comportamento das firmas para o entendimento dos mecanismos de transmissão de política econômica.

Antes de concluirmos esta seção, vale observar que o que foi discutido até o momento referia-se a uma firma individual. Cabe-nos perguntar então: e para a indústria, como interpretar o conceito de capacidade? Claro que o somatório de capacidades das firmas individuais de um setor excederia em muito o produto que poderia ser obtido dentro desse setor. Se todas as firmas tentarem, ao mesmo tempo, produzir a sua capacidade máxima, muito rapidamente se darão conta de que será impossível prosseguir, dada a escassez de matérias-primas, capital e trabalho que advirá. Desta forma, o conceito agregado de capacidade é normalmente referido como sendo o produto potencial de um setor, ou seja, o nível de produto máximo possível, considerando as limitações impostas pela interdependência dos diferentes setores, segundo a estrutura produtiva em determinado momento.

\section{FORMAS DE MENSURAÇÃO}

As dificuldades na avaliação quantitativa de capacidade e grau de utilização de capacidade começam por sua conceituação que, conforme vimos, não apresenta uma interpretação teórica única. ${ }^{10}$ Como conseqüência, a interpretação econômica dessas medidas deve ser qualificada com hipóteses sobre o processo de decisão das firmas, e os resultados obtidos com a aplicação das metodologias existentes são sujeitos a essas qualificações. Há diversas metodologias para se investigar empiricamente o grau de ocupação de capacidade. Abordaremos nesta seção, sem a pretensão de ser exaustivos, uma discussão sobre estimativas obtidas por meio de levantamentos diretos e estimativas obtidas de forma indireta.

\subsection{Estimativas diretas e o conceito de capacidade prática de produção}

As estimativas diretas consistem em pesquisas periódicas junto a empresários sobre sua avaliação quanto ao grau de utilização da capacidade e adições à capacidade já instalada. A vantagem desse método é que as perguntas são respondidas por quem sabe as respostas. Possibilita também obter ou- 
tras informações impossíveis de conseguir por outros métodos, como por exemplo taxas de operação que seriam preferidas, despesas previstas para gastos de capital etc.

As pesquisas diretas variam segundo a maior ou menor precisão na orientação sobre a definição de capacidade. Referência internacional importante é a pesquisa do Bureau of Census, ${ }^{11}$ que no seu levantamento busca precisar o conceito de capacidade, explicitando instruções de como definir o conceito de capacidade prática: "the greateste level of ouptput (...) [the] plant can achieve within the framework of a realistic work pattern". Recomenda-se que o respondente deve considerar para avaliar a capacidade prática:

(a) assumir um mix normal de produtos. Se a planta é sujeita a variações no curto prazo, no mix de produtos deve ser assumido que o padrão corrente de produção é normal;

(b) estabelecer a capacidade em termos do número de turnos e horas de operação da planta considerando uma expansão das operações que sejam razoavelmente obtidas na sua indústria ou localidade;

(c) considerar apenas o maquinário e equipamento em condições de ser operado;

(d) levar em consideração o período de manutenção, reparação e limpeza que será requerido na medida em que a planta se mova da operação corrente para a plena capacidade;

(e) assumir a disponibilidade de mão-de-obra, matérias-primas etc. que sejam suficientes para utilizar o equipamento e o maquinário em operação no período;

(f) desconsiderar os custos adicionais, quer sejam de mão-de-obra, quer sejam de materiais como fatores limitantes ao definir a capacidade instalada;

(g) considerar a utilização de contratação de serviços ou o uso de facilidades de produção fora da planta em condições normais.

No Brasil há algumas experiências de inquéritos estatísticos que realizam estimativas do grau de utilização de capacidade na indústria. A Confederação Nacional da Indústria (CNI), por exemplo, realiza, desde fins dos anos 1990, inquérito mensal junto as indústrias brasileiras levantando, dentre outras variáveis, o grau de utilização de capacidade. A estimativa do grau de ocupação deve ser feita segundo os critérios que a própria empresa usual- 
mente emprega para definir o grau de ocupação da sua capacidade. ${ }^{12}$ Vale observar que a CNI identifica como unidade de investigação a empresa e não a planta industrial, ${ }^{13}$ como no caso citado da pesquisa do Bureau of Census.

Além desses tipos de inquéritos, existem as sondagens conjunturais, cujo objetivo é captar a opinião dos empresários sobre diversos aspectos de sua atividade. No Brasil, a Fundação Getúlio Vargas realiza trimestralmente esse tipo de levantamento desde os anos 1970, baseada na experiência pioneira do Instituto de Pesquisa Econômica de Munique, perguntando aos empresários qual foi, nos últimos três meses, o grau de utilização da capacidade instalada. Nesse tipo de pesquisa não é especificado o que deve ser entendido por capacidade, acreditando-se que o empresário responde algo próximo à capacidade prática.

A dificuldade normalmente apontada na interpretação dos resultados das pesquisas diretas é o fato de não ser claro como os empresários definem capacidade, a despeito das recomendações dadas, com maior ou menor grau de detalhe como exemplificamos acima, nos levantamentos diretos. Segundo Phillips (1963: 285), em pesquisa direta, o risco que se corre é devido ao viés na avaliação dos respondentes sobre a capacidade operacional. Para o autor, esse viés é decorrente do "emotive bias due to the feelings businessmen have".

Mesmo que uma boa estimativa do conceito de capacidade prática de produção seja feita, Christiano (1981) chama atenção para o fato de que algumas dúvidas ainda persistem sobre a interpretação adotada pelo respondente, como, por exemplo, se o equipamento marginal está ou não sendo incluído na estimativa, pois, de acordo com as pressões de demanda, ele pode ou não ser incorporado (nesse caso os informantes estariam declarando seus percentuais de utilização com base numa capacidade que seria a desejada). Outro aspecto é o que diz respeito a setores que produzem diversos produtos e cuja demanda varia muito. Mesmo que se indique um mix normal a ser considerado, é difícil evitar alguma ambigüidade. ${ }^{14}$

Essas questões são, do ponto de vista estatístico, insolúveis, cabendo considerá-las perante as perspectivas de análise teórica que se pretende adotar com os resultados do levantamento.

Em relação aos outros métodos de estimativa (ver item seguinte), as pesquisas diretas, dadas suas características, têm a tendência de mostrar uma 
subtilização de capacidade maior. Algumas das razões para isso, conforme aponta Christiano (op. cit.), são: ${ }^{15}$

(a) firmas com rendimentos crescentes podem construir plantas maiores do que seriam justificadas pela sua participação atual no mercado, uma vez que esperam se expandir no futuro;

(b) flutuações na demanda podem tornar mais racional para a firma manter excesso de capacidade para atender a momentos de pico de demanda;

(c) flutuações no preço dos insumos tornam mais econômico manter a capacidade ociosa em alguns períodos. Exemplo é a remuneração mais elevada do trabalho noturno que justifica a ociosidade da planta durante a noite;

(d) por fim, segundo a teoria da concorrência imperfeita, firmas operando em mercados onde não prevaleçam as regras da concorrência perfeita intencionalmente manterão excesso de capacidade, uma vez que o ponto de maximização de lucro não coincide com o de plena utilização.

Pesquisa conduzida por Corrado e Mattey (1997), baseados em levantamento estatístico do Bureau of Census sobre capacidade, mostra que é possível identificar junto aos informantes o que eles interpretam como capacidade de produção. Segundo os autores, nem todas as plantas industriais classificam os fatores de produção como fixos e variáveis da mesma maneira. Dessa forma, distinguem três formas de definir capacidade, conforme as características técnicas de operação das plantas.

O primeiro tipo que identificam é a linha de operação industrial, onde é possível aumentar a produção fazendo variar o período normal de trabalho. Ou seja, são plantas que aumentam a duração dos turnos de trabalho, especialmente quando enfrentam choques passageiros de demanda, ou adicionam mais um turno quando se deparam com mudanças mais persistentes. Um exemplo desse tipo de planta são as montadoras de veículos. Nesses casos, ao definir o nível de emprego dos fatores de produção, identificam como capacidade plena de produção o período máximo realista pelo qual a planta pode funcionar. O aumento no grau de utilização de capacidade apresenta uma correlação elevada com o aumento nos custos variáveis de produção. 
Um segundo tipo de operação de planta industrial é aquela organizada em operações flexíveis com um conjunto de máquinas fazendo o mesmo serviço. A concepção de capacidade neste caso decorre principalmente do tempo e da intensidade de trabalho em cada máquina. Mudanças nos períodos de trabalho na planta explicam menos a variação na capacidade corrente do que no caso anterior.

O terceiro tipo de planta é a de funcionamento contínuo, ou seja, são plantas que devem operar 24 horas por dia, 7 dias na semana. Um exemplo típico é uma refinaria de petróleo. Plantas com esta tecnologia não param sua produção, exceto em condições muito adversas de demanda. Dessa maneira, quando avaliam sua capacidade, consideram como determinante os serviços de capital dados por um estoque fixo de máquinas e equipamentos. O aumento no grau de utilização de capacidade nesses casos implica incorporar ao processo produtivo mais unidades de produção.

Em resumo, em termos práticos, com base na experiência internacional de levantamentos estatísticos diretos, considera-se que os empresários informam o que se convencionou chamar de capacidade prática de produção. Ou seja, dado que não existe uma forma de se especificar exatamente como medir capacidade, orienta-se o entrevistado para que estime capacidade como o maior produto que a firma/planta poderia alcançar empregando seu equipamento ao máximo dentro de um quadro realista de rotinas de trabalho e assumindo um mix de produtos normal.

\subsection{Relação capital-produto e estimativas derivadas da função de produção}

A estimativa de grau de utilização de capacidade pode ser feita por métodos indiretos, como por exemplo a partir da relação capital-produto. Esse método consiste em estimar a taxa de utilização de capacidade instalada num determinado setor a partir da relação entre o estoque de capital e a produção gerada ao longo dos anos. O menor valor desta série é tomado como indicador de utilização máxima, o qual é usado para estimar o produto potencial. Parte-se da hipótese de que existe uma relação estável entre o estoque de capital e a produção gerada ao longo dos anos e que variações no uso do estoque são uma boa proxy de variação no grau de utilização. 
A maior dificuldade desse tipo de estimativa é o cálculo do estoque de capital, que apresenta inúmeros problemas teóricos e práticos. No Brasil, em particular, em razão do longo período de alta inflação, essas estimativas se tornaram virtualmente impossíveis de serem feitas para os anos 1980, em especial. Uma vantagem desse método em relação às estimativas diretas é o fato de considerar os efeitos de variações no investimento sobre o produto potencial.

Outro exercício para estimar o grau de utilização de capacidade com longa tradição na literatura econômica é o que parte do emprego de funções de produção para estimativas de produto potencial e conseqüentemente o hiato do produto. Nesse caso, o conceito de utilização de capacidade é condicionado ao estoque de capital disponível. Estimativas com base em funções de produção permitem introduzir algumas sofisticações de cálculo como, por exemplo, a separação das contribuições de cada um dos fatores de produção para o crescimento do produto potencial (por exemplo, Klein e Preston, 1967). Deve-se registrar também estudos que se preocupam em captar os efeitos de variações de preços dos insumos sobre a capacidade, em particular de energia (por exemplo, Berndt e Morrison, 1981). Vale observar que essas metodologias são mais empregadas em estudos analíticos do que em análises de conjuntura.

\section{RESUMO E COMENTÁRIOS FINAIS}

A perspectiva de retomada de crescimento da economia brasileira após um longo período de baixa taxa de investimento colocou em evidência a discussão sobre a capacidade do setor produtivo de atender ao aumento da demanda sem pressionar a inflação. Nesse contexto, em acompanhamentos de conjuntura, a evolução do grau de utilização da capacidade pelas firmas assumiu um interesse especial. Em particular, nas atas do COPOM, a evolução do grau de utilização de capacidade tem sido objeto de comentário sistemático.

A questão que nos propomos a debater neste texto é sobre o grau de precisão da medida de utilização de capacidade, considerando que a credibilidade de política econômica também depende das estatísticas utilizadas na argumentação sobre as decisões tomadas. No Brasil, as medidas de grau 
de utilização de capacidade não são produzidas por fontes oficiais, ou seja, não há um controle público sobre a qualidade dos resultados disponíveis.

Assim, nosso objetivo é, a partir do reconhecimento da importância das estimativas de capacidade produtiva, discutir, sem sermos exaustivos, a conceituação teórica de capacidade de produção à luz de diferentes correntes de pensamento econômico e segundo diferentes metodologias estatísticas. Iniciamos nossa discussão com a pesquisa de Robin Marris sobre a economia da utilização de capacidade, em que o autor busca explicar por que as firmas operam com excesso de capacidade. Mostramos que não é possível conhecer objetivamente o limite planejado de capacidade de produção, quando da implementação de planos de investimento. Essa dificuldade na estimativa da capacidade planejada condiciona a interpretação sobre o grau de ocupação da capacidade. Nesse sentido, interpretações são oferecidas sobre o processo de decisão das firmas sobre como ocupar sua capacidade produtiva.

Neste trabalho, exploramos duas argumentações sobre o comportamento da firma. Uma linha de argumentação sobre o que seja capacidade de produção enfatiza aspectos de engenharia, apontando como restrições à utilização máxima da capacidade questões relacionadas à técnica de produção utilizada. Do ponto de vista teórico, essa definição sugere que a firma é apenas uma planta sujeita às leis de rendimento, cuja estratégia é maximizar lucro no curto prazo, alocando eficientemente seus recursos produtivos, dados os preços relativos dos fatores de produção.

Uma outra linha de argumentação considera que a firma, operando em um ambiente de incerteza, estabelece metas de retorno sob um volume padrão de produção. Na prática, fixam seus preços e, em função destes, a quantidade a ser produzida. Destacamos que, nessa perspectiva de comportamento da firma, o importante para se estimar a capacidade disponível e sua utilização é conhecer como as expectativas dos empresários são formadas sobre o comportamento futuro da demanda. Essa é uma interpretação de orientação keynesiana, condizente com o conceito de custo de uso proposto na Teoria Geral.

Do ponto de vista empírico, investigamos os levantamentos diretos sobre capacidade e grau de utilização e estimativas via funções de produção. No primeiro caso, ilustramos, com as instruções do levantamento do Bureau of Census, como empiricamente o conceito de capacidade é elabora- 
do. Estudo de Corrado e Mattey (1997) sobre a forma como a informação de capacidade é informada conclui que, na prática, o conceito investigado de capacidade corresponde ao que se convencionou chamar de capacidade prática de produção. No caso do Brasil, os levantamentos diretos mencionados deixam mais liberdade ao respondente para interpretar o conceito de capacidade prática de produção, possivelmente tornando-o mais impreciso, considerando inclusive que o conceito se refere à empresa, e não a plantas industriais. Levantamentos indiretos no Brasil contam com uma limitação que são as estatísticas de estoque de capital.

Podemos concluir, como Steindl (1952), que qualquer medida de capacidade é uma convenção. Vimos que a literatura econômica reconhece que a conceituação de capacidade é ambígua e, portanto, sua interpretação depende da forma como se supõe que as firmas formam sua expectativa sobre o que seja seu limite de capacidade. Assim, entendemos que não existe uma única interpretação teórica sobre como conceituar capacidade. Em termos empíricos, há uma diversidade de técnicas e formas de levantamentos. A despeito das dificuldades teóricas e operacionais, estatísticas sobre o grau de utilização de recursos produtivos são indispensáveis para o planejamento tanto macroeconômico como microeconômico e, nesse sentido, a qualidade dessas estatísticas bem como as hipóteses implícitas na sua conceituação devem ser objeto de preocupação dos analistas econômicos.

\section{NOTAS}

1. Okun sugere uma metodologia para estimar o produto potencial que consiste em estabelecer uma relação entre variações no nível de emprego e variações no nível de atividade tomando o desemprego como uma medida de quanto de produto potencial está sendo "desperdiçado". Uma crítica à sua metodologia é feita pelo próprio autor que afirma: “(...) measures of excess of capacity in industrial plant and equipment do show a close relationship to unemployment — idle men are accompanied by idle machines. But the correlation is not perfect and operating rates in industry should be considered along with employment data as an indicator of the gap between potential and actual output (...) Still I shall feel happier with the estimation of potential output when (...) the capital factor can be explicitly taken into account" (Okun, op. cit.: 410). No período mais recente o cálculo do produto potencial ganhou relevância na estimativa de funções de reação dos bancos centrais, partindo de uma regra de Taylor na qual o desvio do produto efetivo para o produto potencial é considerado.

2. É importante destacar que não estamos com isso dizendo que não há qualidade na produção de estatísticas de grau de utilização de capacidade por fontes privadas. 
3. Deve-se observar que é referência obrigatória nos relatórios do Comitê de Política Monetária. As fontes estatísticas para mensurar o grau de utilização de capacidade são da pesquisa produzida pela CNI - Indicadores Industriais, e da Sondagem Conjuntural da FGV. Para uma análise do desempenho da indústria em 2004, com informações sobre grau de utilização de capacidade, ver Carvalho e Feijó (2005).

4. Uma apresentação das teses de Marris encontra-se em Baumann Neves (1976). Uma estimativa da medida de utilização de capacidade proposta por Marris para a economia americana, por exemplo, pode ser vista em Foss (1981).

5. "The statistic with which we shall mainly be concerned is the shift work ratio, the proportion of operatives in a plant or industry reported as engaged on shifts". (Marris, op. cit.: 120 , grifos do original).

6. Vale observar que a pesquisa de Marris levantou essas questões.

7. Vale notar que, mesmo no âmbito da teoria convencional, há reconhecimento de que as respostas dos preços não são imediatas às pressões da demanda. O ponto importante é que, mesmo com esse reconhecimento, a firma desempenha um papel passivo no contexto teórico, reagindo mecanicamente a estímulos predefinidos.

8. Podemos citar como uma referência na literatura sobre teoria da firma que se adeqüe a essa interpretação a teoria da firma de Penrose (1980), na qual o que é relevante no processo de tomada de decisão é a percepção do empresário sobre suas capacidades e as oportunidades oferecidas pelo ambiente econômico: 'Embora a oportunidade 'objetiva' da firma seja limitada pelo que a firma é capaz de alcançar, a oportunidade 'subjetiva' é uma questão do que a firma acredita que pode alcançar. 'Expectativas' e não fatos 'objetivos' são os determinantes imediatos do comportamento da firma” (Penrose, 1980: 41). Em outra passagem, "O ambiente tem sido tratado [no seu estudo] não como um fato 'objetivo', mas como uma 'imagem' na mente do empresário: a justificativa para esse procedimento é a hipótese de que não é o ambiente 'como tal', mas sim o ambiente que o empresário vê, que é o relevante para suas ações” (ibid.: 215).

9. Para uma discussão sobre os microfundamentos da oferta agregada com base no custo de uso do capital, ver Chick (1983), capítulo 6.

10. A dificuldade maior ocorre nos casos em que a produção não pode ser inteiramente determinada nem por condições físicas nem por considerações de demanda simplesmente.

11. Ver Bureau of Census (1975).

12. O respondente deve informar "Parcela da capacidade de produção operacional em condições normais de funcionamento utilizada no mês. Deve ser expressa em \%. O valor informado não deverá ultrapassar os $100 \%$ que correspondem à utilização máxima da capacidade instalada. Este percentual a ser informado deverá ser calculado ou estimado pela empresa, segundo os critérios que ela própria usualmente emprega para definir o grau de ocupação da sua capacidade produtiva. Quaisquer mudanças na capacidade de produção instalada devem ser mencionadas utilizando o espaço designado no questionário". (CNI, s/d). 
13. Para uma discussão sobre unidade de investigação estatística em inquéritos estatísticos no Brasil, ver Feijó e Valente (2004).

14. Phillips (op. cit.: 287) afirma que "If output were defined in terms of the product mix existing at the time of the survey, two measures of excess capacity are possible. One is the difference between the actual output rate of the mix and a capacity output rate of the same mix. The other is the difference between the capital stocks being utilized for actual output, and the total capacity stock. When structural factors are significant, the latter would yield a larger estimate for excess capacity than would the former. But if the existing mix reflects market choice for the allocation of the economy's resources, the former seens more relevant".

15. Como ilustração citamos estudo sobre capacidade ociosa na indústria paulista nos anos 1980 que discute o conceito de capacidade nos seguintes termos: "A capacidade produtiva, sob o aspecto técnico, seria a máxima produção possível de ser obtida em condições normais de trabalho. Porém, o que são condições normais? Empresas que trabalham 24 horas por dia, durante $1 / 3$ do ano, por exemplo, indústria de beneficiamento de produtos agrícolas - e permanecem inativas durante os $2 / 3$ restantes estariam operando em condições normais? Em termos de turnos de trabalho, qual seria o padrão normal: 1 turno ou 3 turnos? Como determinar a produção máxima normal para empresas cujas atividades estão vinculadas à moda, a dias festivos, ao clima, etc.? Como determinar a produção máxima para empresas com variada linha de produtos e que estão aptas a alterar a linha com relativa facilidade? O superdimensionamento - inevitável por razões técnicas, digamos - ainda assim caracterizaria uma capacidade ociosa?" (Economia Paulista, 1980: 93).

\section{REFERÊNCIAS BIBLIOGRÁFICAS}

BAUMANN NEVES, R. (1976) “Os ciclos na indústria de transformação: um estudo da utilização da capacidade no Brasil, 1955-1975”. Dissertação de mestrado, UnB.

BERNDT, E. R., MORRISON, C. J. (1967) "Capacity utilization measures underlying economic theory and na alternative approach”. American Economic Review, v. 71 , n. 2, May.

BUREAU OF CENSUS (1975) Survey of Plant Capacity.

Carvalho, P. G. M., Feijó, C. (2005) “A indústria brasileira em 2004 e as teses sobre a sustentabilidade da retomada do crescimento". Indicadores FEE, v. 33, n. 1.

CHICK, V. Macroeconomics after Keynes. MIT Press.

CHRISTIANO, L. (1981) “A survey of measures of capacity utilisation”. IMF Staff Papers, 28 (1).

CNI. Indicadores Industriais CNI: Metodologia, www.cni.org.br.

CORRADO, C., MATTEY, J. (1997) “Capacity utilization”. Journal of Economic Perspective, v. 11, n. 1.

DAVIDSON, P. (1978) Money and the Real World. 2. ed. MacMillan Co. 
ECONOMIA PAULISTA (1980) “A capacidade ociosa na indústria”. Revista da Secretaria de Fazenda, fev.

FEIJÓ, C. A., VALENTE, E. (2004) “A firma na teoria econômica e como unidade de investigação estatística: evolução nas conceituações”. Revista de Economia Contemporânea, v. 8, n. 2.

FOSS, M. F. (1981) "Long run changes in the work week of fixed capital”. American Economic Review, v. 71, n. 2, May.

KEYNES, J. M. (1936) The General Theory of Employment Interest and Money. MacMillan, Cambridge University Press.

LEEUW, F. (1962) “The concept of capacity”. Journal of American Statistical Association, 57 (300).

MARRIS, R. (1964) The Economics of Capital Utilisation. Cambridge University Press.

OKUN, A. (1962) "Potential GNP: Its measurement and significance”. In: M. G. Mueller (ed.), Reading in Macroeconomics. 2. ed.

PENROSE, E. T. (1959 e 1980) The Theory of the Growth of the Firm. Nova York: John Wiley.

PHILLIPS, A. (1963) “An appraisal of measures of capacity”. American Economic Review, v. 53, n. 1 .

STEINDL, J. (1952) Maturity and Stagnation of American Capitalism. Oxford University Press. 